\title{
A Normal (0, 1)-Polytope None of Whose Regular Triangulations Is Unimodular*
}

\author{
H. Ohsugi ${ }^{1}$ and T. Hibi ${ }^{2}$ \\ ${ }^{1}$ Department of Mathematics, \\ Graduate School of Science, \\ Osaka University, \\ Toyonaka, Osaka 560, Japan \\ smv067oh@ex.ecip.osaka-u.ac.jp \\ ${ }^{2}$ Department of Mathematics, \\ Graduate School of Science, \\ Osaka University, \\ Toyonaka, Osaka 560, Japan \\ hibi@math.sci.osaka-u.ac.jp
}

\begin{abstract}
A normal (0,1)-polytope none of whose regular triangulations is unimodular is constructed.
\end{abstract}

Let $\mathcal{P} \subset \mathbb{R}^{N}$ be an integral convex polytope, i.e., a convex polytope any of whose vertices has integer coordinates. Let $K\left[t_{1}, t_{1}^{-1}, \ldots, t_{N}, t_{N}^{-1}, s\right]$ denote the Laurent polynomial ring over a field $K$. We write $K[\mathcal{P}]$ for the subalgebra of $K\left[t_{1}, t_{1}^{-1}, \ldots, t_{N}, t_{N}^{-1}, s\right]$ which is generated by all monomials $t_{1}^{\alpha_{1}} \cdots t_{N}^{\alpha_{N}} s$ with $\left(\alpha_{1}, \ldots, \alpha_{N}\right) \in \mathcal{P} \cap \mathbb{Z}^{N}$. An integral convex polytope $\mathcal{P}$ is called normal if the subalgebra $K[\mathcal{P}]$ is normal, i.e., integrally closed in its quotient field. We are interested in the combinatorial properties of integral convex polytopes as follows:

(i) $\mathcal{P}$ possesses a unimodular regular triangulation;

(ii) $\mathcal{P}$ possesses a unimodular triangulation;

(iii) $\mathcal{P}$ possesses a unimodular covering; and

(iv) $\mathcal{P}$ is normal.

We then have the hierarchy (i) $\Rightarrow$ (ii) $\Rightarrow$ (iii) $\Rightarrow$ (iv). How about the converse of each of

\footnotetext{
* This research was performed while the second author was staying at the Mathematical Sciences Research Institute in Berkeley, February-April, 1997, supported in part by NSF Grant \#DMS 9022140.
} 
the above three " $\Rightarrow$ "? First, no counterexample of an integral polytope is known for the converse of (i) $\Rightarrow$ (ii). Second, there exist many integral polytopes which disprove the converse of (ii) $\Rightarrow$ (iii). However, we do not know a $(0,1)$-polytope which disproves the converse of (ii) $\Rightarrow$ (iii). Third, no counterexample of an integral polytope is known for the converse of (iii) $\Rightarrow$ (iv).

The purpose of the present paper is to give the first normal $(0,1)$-polytope which possesses no unimodular regular triangulation. We refer the reader to, e.g., [2] for fundamental definitions and results on, e.g., initial ideals, toric ideals, Gröbner bases, and regular triangulations.

Let $G$ be a finite connected graph on the vertex set $V(G)=\{1, \ldots, d\}$ having no loop and no multiple edge. If $e=\{i, j\}$ is an edge of $G$ joining $i \in V(G)$ with $j \in V(G)$, then we define $\rho(e) \in \mathbb{R}^{d}$ by $\rho(e)=\mathbf{e}_{i}+\mathbf{e}_{j}$. Here, $\mathbf{e}_{i}$ is the $i$ th unit coordinate vector in $\mathbb{R}^{d}$. We write $\mathcal{P}_{G} \subset \mathbb{R}^{d}$ for the convex hull of the finite set $\{\rho(e) ; e$ is an edge of $G\} \subset \mathbb{R}^{d}$ and call $\mathcal{P}_{G}$ the edge polytope of $G$. It is known, e.g., [1, Corollary 2.3] that $\mathcal{P}_{G}$ is normal if and only if, for arbitrary two odd cycles $C$ and $C^{\prime}$ in $G$ having no common vertex, there exists an edge of $G$ joining a vertex of $C$ with a vertex of $C^{\prime}$. It follows from [2, Corollary 8.9] that no regular triangulation of $\mathcal{P}_{G}$ is unimodular if and only if no initial ideal of the toric ideal $I_{G}$ of $K\left[\mathcal{P}_{G}\right]$ is square-free.

Throughout the remainder of the paper, let $G$ denote the finite connected graph with 10 vertices and 15 edges below.

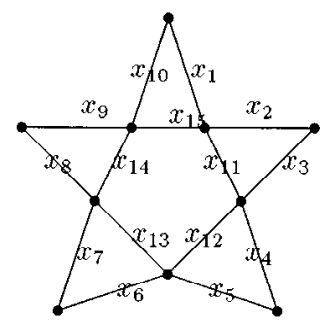

Then, $\operatorname{dim} \mathcal{P}_{G}=9$ and the normalized volume of $\mathcal{P}_{G}$ is equal to 56 . By virtue of the above combinatorial criterion for the normality of edge polytopes, we easily see that $\mathcal{P}_{G}$ is normal. Now, in order to show that no initial ideal of $I_{G}$ is square-free, we first compute the Graver basis of $I_{G}$ by [2, Algorithm 7.2]:

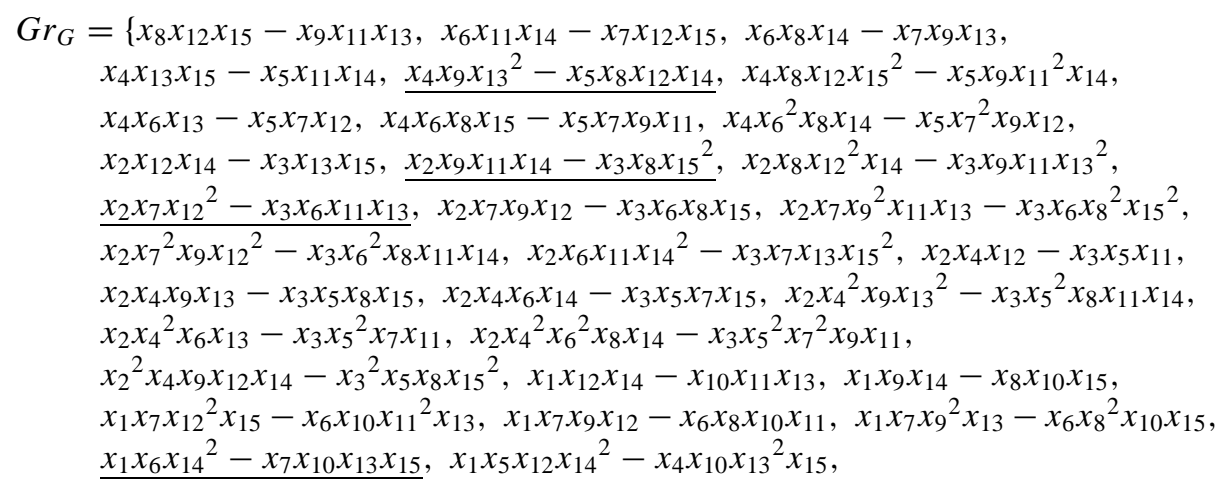




$$
\begin{aligned}
& x_{1} x_{5} x_{7}^{2} x_{9}^{2} x_{12}-x_{4} x_{6}{ }^{2} x_{8}{ }^{2} x_{10} x_{15}, x_{1} x_{4} x_{12} x_{15}-x_{5} x_{10} x_{11}^{2}, \\
& x_{1} x_{4} x_{9} x_{13}-x_{5} x_{8} x_{10} x_{11}, x_{1} x_{4} x_{9}{ }^{2} x_{13}{ }^{2}-x_{5} x_{8}{ }^{2} x_{10} x_{12} x_{15}, x_{1} x_{4} x_{6} x_{14}-x_{5} x_{7} x_{10} x_{11} \text {, } \\
& x_{1} x_{4} x_{6}{ }^{2} x_{14}{ }^{2}-x_{5} x_{7}^{2} x_{10} x_{12} x_{15}, x_{1} x_{4}{ }^{2} x_{6} x_{13} x_{15}-x_{5}{ }^{2} x_{7} x_{10} x_{11}{ }^{2} \text {, } \\
& x_{1} x_{3} x_{15}-x_{2} x_{10} x_{11}, x_{1} x_{3} x_{9} x_{13}-x_{2} x_{8} x_{10} x_{12} \text {, } \\
& x_{1} x_{3} x_{6} x_{14}-x_{2} x_{7} x_{10} x_{12}, x_{1} x_{3} x_{5} x_{14}-x_{2} x_{4} x_{10} x_{13} \text {, } \\
& x_{1} x_{3} x_{5} x_{7} x_{9}-x_{2} x_{4} x_{6} x_{8} x_{10}, x_{1} x_{3}^{2} x_{6} x_{13} x_{15}-x_{2}^{2} x_{7} x_{10} x_{12}{ }^{2} \text {, } \\
& x_{1} x_{3}{ }^{2} x_{5} x_{15}-x_{2}{ }^{2} x_{4} x_{10} x_{12}, x_{1} x_{3}{ }^{2} x_{5}{ }^{2} x_{7} x_{15}-x_{2}{ }^{2} x_{4}{ }^{2} x_{6} x_{10} x_{13} \text {, } \\
& x_{1}^{2} x_{4} x_{9} x_{12} x_{14}-x_{5} x_{8} x_{10}^{2} x_{11}^{2}, x_{1}^{2} x_{3} x_{9} x_{14}-x_{2} x_{8} x_{10}^{2} x_{11} \text {, } \\
& x_{1}^{2} x_{3} x_{7} x_{9}^{2} x_{13}-x_{2} x_{6} x_{8}{ }^{2} x_{10}{ }^{2} x_{11}, x_{1}{ }^{2} x_{3} x_{6} x_{14}{ }^{2}-x_{2} x_{7} x_{10}{ }^{2} x_{11} x_{13} \text {, } \\
& \left.x_{1}^{2} x_{3}^{2} x_{5} x_{9} x_{14}-x_{2}^{2} x_{4} x_{8} x_{10}^{2} x_{12}\right\} \text {. }
\end{aligned}
$$

It follows that each of the two terms of each of the above five underlined binomials can be divided by no term of the binomials belonging to $G r_{G}$. Hence, with respect to an arbitrary term order, each of the five underlined binomials can be reduced by no binomial belonging to $G r_{G}$. Since, in general, the Graver basis contains the universal Gröbner basis, every reduced Gröbner basis of $I_{G}$ is a subset of $G r_{G}$. Thus, with respect to an arbitrary term order, all of the five underlined binomials must appear in the reduced Gröbner basis of $I_{G}$. Hence, the toric ideal $I_{G}$ has a square-free initial ideal only if we can find a term order $\succ$ such that all of the initial terms of the five underlined binomials are simultaneously square-free. Now, suppose that there exists such a term order $\succ$ and choose a nonnegative weight vector $\omega=\left(\omega_{1}, \ldots, \omega_{15}\right)$ which represents $\succ$. Since the initial terms of the five underlined binomials are simultaneously square-free, the weight vector $\omega$ satisfies the following five simultaneous inequalities:

$$
\left\{\begin{array}{l}
\omega_{5}+\omega_{8}+\omega_{12}+\omega_{14}>\omega_{4}+\omega_{9}+2 \omega_{13}, \\
\omega_{2}+\omega_{9}+\omega_{11}+\omega_{14}>\omega_{3}+\omega_{8}+2 \omega_{15} \\
\omega_{3}+\omega_{6}+\omega_{11}+\omega_{13}>\omega_{2}+\omega_{7}+2 \omega_{12} \\
\omega_{7}+\omega_{10}+\omega_{13}+\omega_{15}>\omega_{1}+\omega_{6}+2 \omega_{14} \\
\omega_{1}+\omega_{4}+\omega_{12}+\omega_{15}>\omega_{5}+\omega_{10}+2 \omega_{11}
\end{array}\right.
$$

Since the sum of the left-hand sides of the five inequalities is equal to that of the righthand sides, the above simultaneous inequalities have no solution in $\mathbb{R}^{15}$. Hence, there exists no term order such that all of the initial terms of the five underlined binomials in $G r_{G}$ are simultaneously square-free. Thus, no initial ideal of the toric ideal $I_{G}$ can be square-free.

In [1] we show that all normal edge polytopes $\mathcal{P}_{G}$ of finite graphs $G$ possess unimodular coverings.

Remark. The edge polytope $\mathcal{P}_{G}$ discussed above does possess a unimodular triangulation. This fact was first verified by Firla and Ziegler by means of an integer programming approach. Hence, this edge polytope yields the only known counterexample for the converse of (i) $\Rightarrow$ (ii). No other example of an integral polytope which possesses a unimodular triangulation and which possesses no unimodular regular triangulation is known. Moreover, an analysis by De Loera (using his program PUNTOS) shows even stranger behavior of this edge polytope: Neither a triangulation with the maximal number 
of simplices nor a triangulation with the minimal number of simplices is achieved by a regular triangulation.

We are grateful to the referee for pointing out the above remark.

\section{References}

1. H. Ohsugi and T. Hibi, Normal polytopes arising from finite graphs, Preprint (March, 1997).

2. B. Sturmfels, Gröbner Bases and Convex Polytopes, American Mathematical Society, Providence, RI, 1995.

Received April 1, 1997, and in revised form June 20, 1997. 